\title{
Calcitonina elevata e gozzo nodulare: che fare?
}

\author{
Antonio Matrone $^{1} \cdot$ Eleonora Molinaro $^{1}$
}

Pubblicato online: 13 luglio 2020

(c) The Author(s) 2020

\section{Commento al weekendo n. 89}

Il quiz n. 89 (http://societaitalianadiendocrinologia.it/public// pdf/quiz89.pdf) "Una calcitonina in fuorigioco" si riferiva a una donna di 46 anni, ipertesa, affetta da sindrome di Steinert e da un piccolo gozzo multinodulare con il nodulo di maggiori dimensioni già definito Tir 2 alla FNA, con calcitonina elevata $(17,9 \mathrm{pg} / \mathrm{ml})$ che si raddoppiava dopo due mesi e che risultava $120 \mathrm{pg} / \mathrm{ml}$ nel liquido di lavaggio della punta dell'ago a un successivo FNA. Al quesito "Nel sospetto di un CMT come procedereste nell'iter diagnostico di questa paziente al fine di programmare il più adeguato trattamento?" meno della metà dei solutori ha indicato la risposta suggerita come corretta e cioè: "Test di stimolo con calcio gluconato con prelievi per CT ai 0', 2', 5', 10', 15 "', mentre la metà dei solutori indicava la risposta n. 1 : "Nessun ulteriore test: $i$ dati raccolti sono sufficienti per inviare la paziente alla chirurgia" (oltre il 22\%) e la risposta n. 4 "Screening genetico per ricerca di mutazioni del protooncogene RET" (oltre il 22\%) e solo una piccola minoranza proponeva il dosaggio del CEA (risposta n. 2).

Il carcinoma midollare della tiroide (CMT) è un tumore neuroendocrino (ad origine dalle cellule parafollicolari o cellule C), con una prevalenza che varia fra il 5 e il $10 \%$ di tutti i tumori tiroidei e dallo 0,4 all' $1,4 \%$ nei noduli tiroidei. Caratteristica peculiare del CMT è quella di essere un tumore ben differenziato, in cui le cellule mantengono intatta la capacità di secernere la calcitonina $(\mathrm{CT})$. Per questo motivo la CT rappresenta un marker altamente sensibile e specifico per la diagnosi di CMT; pochissimi sono, infatti, i casi descritti di CMT calcitonina-negativi [1].

$\bowtie$ A. Matrone

anto.matrone@yahoo.com

1 Dipartimento di Medicina Clinica e Sperimentale, Unità di Endocrinologia 1, Università di Pisa, Pisa, Italia
Mentre la CT è molto utile nella diagnosi preoperatoria, il dosaggio del CEA, invece, non fornisce informazioni utili prima della chirurgia, mentre rappresenta un valido marcatore per valutare l'andamento di malattia dopo la chirurgia, in particolar modo nei casi avanzati.

Sebbene nelle principali linee guida di consenso internazionale non venga espressa chiaramente un'indicazione a favore o contro, il dosaggio routinario della CT sierica nei pazienti con noduli tiroidei è stato associato a una diagnosi precoce e a un miglior decorso ed esito in termini di sopravvivenza malattia specifica [2].

A differenza delle forme differenziate di carcinoma tiroideo, dove l'esame citologico su agoaspirato (FNAC) del nodulo sospetto risulta fondamentale per una corretta diagnosi, la sensibilità di FNAC nella diagnosi di CMT è di circa il 60\%, che indica una discreta imprecisione nella diagnosi stessa [3]. L'utilizzo del dosaggio della CT nel liquido di lavaggio di FNAC sicuramente incrementa la percentuale di diagnosi corrette [4] presentando, però, un problema di fondo rappresentato dalla mancanza di cut-off precisi per la diagnosi di CMT. Inoltre, diversi lavori in letteratura hanno mostrato la superiorità del dosaggio della $\mathrm{CT}$ sierica rispetto a FNAC nella diagnosi di CMT [5].

Anche il dosaggio della CT non è scevro da insidie. Talvolta possiamo trovarci di fronte alla presenza di falsi positivi, che possono essere classificati come "correlati" o "non correlati" a una patologia tiroidea. I falsi positivi "non correlati" sono rappresentati principalmente da: tumori neuroendocrini non-CMT, carcinoma polmonare a piccole cellule, sindrome di Zollinger-Ellison, insufficienza renale cronica, pancreatite, anemia perniciosa, pseudoipoparatiroidismo di tipo I, nonché dall'utilizzo di farmaci come gli inibitori di pompa protonica. Quelli invece "correlati" sono condizioni generalmente associate a un'iperplasia delle cellule $\mathrm{C}$ e rappresentati da: tiroidite linfocitaria cronica e microcarcino- 
ma papillare. Anche la presenza di anticorpi eterofili, seppur meno frequentemente dopo l'introduzione delle nuove metodiche di dosaggio, può determinare risultati falsamente positivi.

Nei casi dubbi, come nel nostro caso clinico con la presenza di valori di CT già ripetuti nel tempo e risultati superiori al limite massimo della norma secondo la metodica di dosaggio, ma non così elevati da permettere una diagnosi precisa di CMT $(<100 \mathrm{pg} / \mathrm{ml})$, il test di stimolo con calcio per CT dovrebbe sempre essere eseguito.

Il razionale dell'utilizzo dello stimolo con calcio risiede nel fatto che le cellule parafollicolari esprimono il calcium sensing receptor (CaSR) a livello extracellulare; tramite questo, riescono a percepire le variazioni di calcio extracellulare e stimolare il rilascio di CT in risposta ad aumenti rapidi del calcio ionizzato.

L'utilizzo del test di stimolo con calcio per CT è utile per 3 motivi:

1. consente di escludere i casi di falsi positivi, in cui la risposta allo stimolo è in genere minima o assente, permettendo un miglior orientamento diagnostico;

2. consente di confermare il sospetto clinico nei casi in cui la CT stimolata superi i valori soglia attualmente disponibili per la diagnosi di CMT;

3. in caso di valori di calcitonina molto elevati dopo lo stimolo, suggerisce ulteriori approfondimenti diagnostici, generalmente tramite metodiche di diagnostica per immagini di secondo livello (TC, RMN) al fine di pianificare l'estensione dell'intervento chirurgico.

La procedura per lo svolgimento del test è relativamente semplice; il test si esegue, infatti, praticando un'infusione e.v. di $2-2,5 \mathrm{mg} / \mathrm{kg}$ di peso corporeo di calcio elemento in $50 \mathrm{ml}$ di soluzione fisiologica $(\mathrm{NaCl} 0,9 \%)$ in 5-10 minuti. La CT viene dosata al tempo 0 prima dell'infusione e dopo 2, 5, 10 e 15 minuti dal termine dell'infusione.

Nel calcolo della quantità di calcio gluconato da infondere bisognerebbe tener conto non solo del peso reale ma, in alcuni casi, soprattutto del peso ideale (http://www. manuelsweb.com/IBW.htm) evitando, così, il rischio di sovradosaggio nei soggetti obesi.

In considerazione della capacità del calcio di aumentare la contrattilità cardiaca, potendo determinare picchi ipertensivi e bradicardia, i pazienti da sottoporre al test dovrebbero eseguire un'attenta anamnesi, in particolar modo di tipo cardiologico (cardiopatie pregresse o in atto, pregresse crisi ipertensive o bradicardie, utilizzo di cardiocinetici). In casi selezionati il test può essere eseguito sotto monitoraggio continuo elettrocardiografico. Nella nostra esperienza, che è costituita dall'esecuzione di più di 250 test al calcio/anno, non abbiamo mai riscontrato eventi avversi di tipo cardiologico né di altro tipo.
L'interpretazione dei risultati del test al calcio è attualmente basata su alcuni lavori presenti in letteratura che hanno cercato di stabilire un cut-off di calcitonina stimolata diagnostico per CMT, differente fra maschi $(>544 \mathrm{pg} / \mathrm{ml}$; sensibilità $77,8 \%$ e specificità $85,4 \%)$ e femmine (>68 pg/ml; sensibilità $83,3 \%$ e specificità $100 \%$ ) [6].

In un paziente con un sospetto di CMT apparentemente sporadico, le indagini genetiche per la ricerca delle mutazioni germinali del gene RET, al fine di identificare i casi familiari (circa il 20\% di tutti i CMT), dovrebbero essere sempre guidate dall'anamnesi familiare e, comunque, posticipate dopo la chirurgia al momento della conferma istologica.

In conclusione, in un paziente con noduli tiroidei in cui il valore della $\mathrm{CT}$ sierica risulti in più determinazioni moderatamente elevato ma non dirimente per la diagnosi di CMT, il test di stimolo con calcio per CT dovrebbe essere sempre eseguito al fine di confermare il sospetto diagnostico. Il test è di facile esecuzione, non necessita di ricovero ospedaliero e risulta privo di significativi eventi avversi.

Ringraziamenti Open access funding provided by Università di Pisa within the CRUI-CARE Agreement.

Conflitto di interesse Gli autori Antonio Matrone e Eleonora Molinaro dichiarano di non avere conflitti di interesse.

Consenso informato Lo studio presentato in questo articolo non ha richiesto sperimentazione umana.

Studi sugli animali Gli autori di questo articolo non hanno eseguito studi sugli animali.

Nota della casa editrice Springer Nature rimane neutrale in riguardo alle rivendicazioni giurisdizionali nelle mappe pubblicate e nelle affiliazioni istituzionali.

Open Access This article is licensed under a Creative Commons Attribution 4.0 International License, which permits use, sharing, adaptation, distribution and reproduction in any medium or format, as long as you give appropriate credit to the original author(s) and the source, provide a link to the Creative Commons licence, and indicate if changes were made. The images or other third party material in this article are included in the article's Creative Commons licence, unless indicated otherwise in a credit line to the material. If material is not included in the article's Creative Commons licence and your intended use is not permitted by statutory regulation or exceeds the permitted use, you will need to obtain permission directly from the copyright holder. To view a copy of this licence, visit http://creativecommons.org/licenses/by/4.0/.

\section{Bibliografia}

1. Sama MT, Rossetto Giaccherino R, Gallo M et al (2016) Clinical challenges with calcitonin-negative medullary thyroid carcinoma. J Cancer Res Clin Oncol 142(9):2023-2029

2. Elisei R, Bottici V, Luchetti F et al (2004) Impact of routine measurement of serum calcitonin on the diagnosis and outcome of medullary thyroid cancer: experience in 10,864 patients with nodular thyroid disorders. J Clin Endocrinol Metab 89(1):163-168 
3. Bugalho MJ, Santos JR, Sobrinho L (2005) Preoperative diagnosis of medullary thyroid carcinoma: fine needle aspiration cytology as compared with serum calcitonin measurement. J Surg Oncol 91(1):56-60

4. Boi F, Maurelli I, Pinna G et al (2007) Calcitonin measurement in wash-out fluid from fine needle aspiration of neck masses in patients with primary and metastatic medullary thyroid carcinoma. J Clin Endocrinol Metab 92(6):2115-2118
5. Elisei R (2008) Routine serum calcitonin measurement in the evaluation of thyroid nodules. Best Pract Res Clin Endocrinol Metab 22(6):941-953

6. Mian C, Perrino M, Colombo C et al (2014) Refining calcium test for the diagnosis of medullary thyroid cancer: cutoffs, procedures, and safety. J Clin Endocrinol Metab 99(5):1656-1664 